\title{
The Effect of Prospective Payment on Admission and Treatment Policy: Evidence from Inpatient Rehabilitation Facilities
}

\author{
Neeraj Sooda,b,c, ${ }^{*}$, Peter J Huckfeldt ${ }^{b}$, David C Grabowskic, ${ }^{\mathrm{d}}$, Joseph P Newhouse ${ }^{\mathrm{c}, \mathrm{d}}$, and \\ José J Escarce ${ }^{b, c, e}$ \\ aUniversity of Southern California (USC), Los Angeles, California \\ ${ }^{b}$ RAND Corporation, Santa Monica, California \\ 'National Bureau of Economic Research (NBER), Cambridge, Massachusetts \\ dHarvard University, Boston, Massachusetts \\ eUniversity of California Los Angeles, Los Angeles, California
}

\begin{abstract}
We examine provider responses to the Medicare inpatient rehabilitation facility (IRF) prospective payment system (PPS), which simultaneously reduced marginal reimbursement and increased average reimbursement. IRFs could respond to the PPS by changing the number of patients admitted, admitting different types of patients, or changing the intensity of care. We use Medicare claims data to separately estimate each type of provider response. We also examine changes in patient outcomes and spillover effects on other post-acute care providers. We find that costs of care initially fell following the PPS, which we attribute to changes in treatment decisions rather than the characteristics of patients admitted to IRFs within the diagnostic categories we examine. However, the probability of admission to IRFs increased after the PPS due to the expanded admission policies of providers. We find modest spillover effects in other post-acute settings and negative health impacts for only one of three diagnostic groups studied.
\end{abstract}

\section{Keywords}

Medicare; treatment intensity; selection; cream skimming; mortality; prospective payment

\section{Introduction}

Concerns over the financial solvency of Medicare have focused attention on curtailing health care spending growth for Medicare beneficiaries. However, these concerns are not new, and the Medicare program has attempted to curtail cost growth for the past several decades. Perhaps the most important, and certainly the most used, weapon in Medicare's arsenal for lowering cost growth has been to change the method Medicare uses to reimburse health care providers. The first such major attempt at cost containment was the 1983 implementation of the acute care hospital prospective payment system. The hope was that

\footnotetext{
(C) 2013 Elsevier B.V. All rights reserved.

"Corresponding Author. University of Southern California, 3335 S. Figueroa St., Unit A, University Park Campus, UGW-Unit A, Los Angeles, California, 90089-7273. Tel: 2138217949 Fax: 2137403460 nsood@ usc.edu.

Publisher's Disclaimer: This is a PDF file of an unedited manuscript that has been accepted for publication. As a service to our customers we are providing this early version of the manuscript. The manuscript will undergo copyediting, typesetting, and review of the resulting proof before it is published in its final citable form. Please note that during the production process errors may be discovered which could affect the content, and all legal disclaimers that apply to the journal pertain.
} 
shifting providers from a cost-based reimbursement to prospectively defined payments would decrease cost growth. Early evidence suggested that the new payment system caused a once-and-for-all reduction in both costs and average acute length of stay at the affected hospitals. However, rates of readmissions increased and care shifted to hospitals that were exempt from prospective payment as well as to hospital outpatient departments and postacute care facilities that continued to be cost reimbursed (Cutler 1995; Kahn et al. 1990; Newhouse 2002; Newhouse and Byrne 1988).

The exemption of post-acute care facilities from prospective payment led to an explosion in both the number and use of post-acute care facilities in the late 1980s and 1990s (Newhouse 2002). Medicare responded again to this dramatic increase in costs by using its weapon of choice: prospective payment. Between 1998 and 2003, prospective payment systems were implemented for each type of post-acute setting: home health agencies; skilled nursing facilities; inpatient rehabilitation facilities; and long-term care hospitals.

More recently, passage of the Affordable Care Act has generated interest in a new approach to prospective payment that provides a single "bundled" payment for an episode of care consisting of an acute-care hospitalization and any post-acute care that occurs within a defined period of time. The hope is that this new payment system can tackle the thorny problem of high Medicare costs without diminishing quality of care. In this context, analyzing past experience with prospective payment for post-acute care and understanding its potential effects is important from both an academic and policy perspective.

In this paper, we estimate the effects of the Medicare inpatient rehabilitation prospective payment system (IRF-PPS). Under the IRF-PPS, an IRF receives a payment for providing care to a patient that is based on a "case-mix group", defined by a patient's age, diagnosis, comorbidities, and functional status. By decreasing marginal reimbursement for additional services, the IRF-PPS was meant to reduce low value services and lead to a more efficient provision of post-acute care. By determining payment as a function of a facility's case mix and increasing reimbursement for more critical patients, the PPS was meant to improve access to IRF care and discourage selective admission of less severe patients (Buntin et al. 2006; Stineman 2002). Increased average payment for patients treated at IRFs thereby mitigated the incentives to curtail costs inherent in reduced marginal payments (Sood, Buntin, and Escarce 2008).

We use the experience with the IRF-PPS to understand how a change in payment policy that simultaneously reduces marginal reimbursement and increases average reimbursement can influence provider behavior. We are not only interested in understanding the effect of this payment change on costs but also in understanding the pathways through which changes might occur. Changes in payments can influence costs in two ways: first, by affecting the "admission policies" or "admission thresholds" of IRFs, that is, the likelihood that a particular patient is admitted to an IRF, and second by affecting the "treatment policies" of IRFs, that is, the intensity of care and type of treatment that a particular patient receives. Moreover, both types of payment changes may have important spillovers for other postacute care providers because of the substitutability of care across sites (Buntin et al. 2010). Finally, prospective payment might have deleterious effects on access to or outcomes of post-acute care if facilities respond to prospective payment incentives by either cream skimming or stinting on care (Ellis and McGuire 1996; Rogers et al. 1990).

In this study, we use a consistent empirical framework and a unique and rich data set to estimate these complex and varied effects of the IRF-PPS. Past empirical research on the IRF-PPS and other prospective payment systems, while contributing to our understanding of the impacts of prospective payment, does not simultaneously evaluate all of the potential 
changes in provider behavior stemming from payment changes (e.g. McCall et al. (2003a; 2003b), McKnight (2006), White (2003)). In some cases, authors estimate effects conditional on using a site of care and do not take a Medicare-wide perspective in analyzing costs, payments and other outcomes of care (e.g. Sood et al. (2008)).

We show that the IRF-PPS led to a substantial increase in payments to IRFs. For example, in the first quarter of 2001, Medicare spent approximately $\$ 11,000$ per IRF patient with a diagnosis of hip fracture. By the full phase-in of the IRF-PPS in the first quarter of 2003, Medicare spent over 25 percent more on IRF care. Our results suggest that this increase in payments led to an increase in the probability of using IRFs for the diagnostic groups examined in our analysis, and that the increase in admission probability was primarily driven by changes in admission policies of IRFs rather than changes in composition of patients being discharged from acute care hospitals. Despite more generous reimbursement for providers, patients received less intensive care once they were admitted to IRFs after the PPS. Again, this primarily reflects changes in treatment policy of IRFs rather than changes in the types of patients admitted to IRFs within the diagnostic categories we examine. Finally, some of the cost savings from reduced intensity of care in IRFs were offset by increased costs at other post-acute care settings. The various changes in patterns of postacute care, however, did not substantively affect mortality, institutionalization, or acute readmissions for two out of the three conditions analyzed in this paper.

The rest of the paper proceeds as follows. In section 2, we discuss the IRF-PPS in greater detail. Section 3 describes the conceptual framework, section 4 data, section 5 our empirical strategy, and section 6 presents results. Section 7 concludes.

\section{The IRF-PPS}

IRFs are inpatient post-acute care sites that provide rehabilitative therapy, either in a hospital-based unit or as a freestanding provider. In 2011, over 300,000 Medicare FFS patients received IRF care, with Medicare payments of $\$ 6.7$ billion dollars (Medicare Payment Advisory Commission 2012). An important factor distinguishing IRFs from other post-acute settings is the intensity of rehabilitation. Medicare requires that patients in IRFs receive 3 hours of therapy per day and mandates that 75 percent of IRFs' patients must be among a set of conditions deemed sufficiently complicated to require such an intense level of therapy.

Prior to the IRF-PPS in January 2002, IRFs were reimbursed based on average costs per discharge, up to a facility-specific limit based on a facility's average costs in its base year of operation; Medicare margins of IRFs ranged from $1.1 \%$ to $1.5 \%$ in the three years prior to the IRF-PPS (Medicare Payment Advisory Commission 2006; Sood et al. 2008). Following the enactment of the IRF-PPS, IRF payment shifted to a prospectively predetermined payment amount per discharge. This payment is a function of the patient's case-mix group (defined by the primary reason for rehabilitation, functional status, age, and comorbidities) and the provider's wage index and other characteristics, and is adjusted for rural status, share of low-income patients, and short-stay and high-cost outliers (Medicare Payment Advisory Commission 2011a). Each IRF transitioned to the PPS in its first fiscal year starting after January 1, 2002. Because of variation in fiscal year start dates, the PPS was effectively phased in throughout 2002, with the transition completed by January 2003 .

\section{Conceptual framework}

The conceptual model for the paper is adapted from Hodgkin and McGuire (1994) and Ellis and McGuire (1996). Hodgkin and McGuire examine the effects of payment change on the intensity of treatment (the "treatment policy"). We model both the decision of the provider 
to admit patients (the "admission policy") as well as decisions regarding treatment intensity (the "treatment policy").

Consider a provider who has $\mathrm{N}$ potential patients eligible for admission into its facility ${ }^{1}$. For each patient, the provider needs to decide the probability of admitting that patient $\left(p_{\mathrm{j}}\right)$ as well as the intensity of care $\left(c_{\mathrm{j}}\right)$ provided to the patient once admitted. We assume a general objective function for the provider where the provider derives utility from profit, the probabilities of admitting patients and the intensity of care provided to patients. The probabilities of admitting patients and the intensity of care enter the utility function directly as health care providers might have altruistic concerns about the welfare or health of eligible patients. The provider faces a mixed payment system with an average reimbursement of $a_{j}$ and a marginal reimbursement of $\mathrm{m}_{\mathrm{j}}$.

First consider the providers' admission problem:

$$
\underbrace{\operatorname{Max}}_{\text {wrt } p_{j}} U\left(E(\pi), p_{j}, c_{j}\right) \text { subject to } E(\pi)=\sum_{j=1}^{N} p_{j}\left(a_{j}+\left(m_{j}-1\right) c_{j}\right)
$$

Where $a_{j}$ is the average reimbursement and $m_{j}$ is the marginal reimbursement, which is a function of observed costs, $p_{\mathrm{j}}$ is the probability of admitting patient $j$ and $c_{\mathrm{j}}$ is the intensity of treatment or the costs of treating patient $j$. Thus, $\left(a_{j}+\left(m_{j}-1\right) c_{j}\right)$ is the profit from patient $j$ The first-order condition for admission policy is:

$$
\left[p_{j}\right]: U_{p_{j}}+U_{\pi}\left(a_{j}+\left(m_{j}-1\right) c_{j}\right)=0
$$

The above condition shows that providers choose the probability of admission so that the marginal utility of increase in admission probability for a particular patient equals the change in profit from admitting the patient times the marginal utility of profit. Also note that if the patient is profitable, $\left(a_{j}+\left(m_{j}-1\right) c_{j}\right)>0$, the probability of admission is one. In other words, all profitable patients will be admitted, assuming no capacity constraints. If there are such constraints, the provider takes the patients that maximize the objective function until the constraint is binding.

We are interested in understanding how provider decisions with regard to admissions change as average $\left(a_{j}\right)$ and marginal $\left(m_{j}\right)$ reimbursement change. An increase in either type of reimbursement increases the expected profitability of patients. Assuming $\left(a_{j}+\left(m_{j}-1\right) c_{j}\right)$ is positive, this makes the second term in right hand side of (2.) larger, thus requiring $U_{p_{j}}$ to fall to maintain the equality, and hence increasing the probability of admission and the total volume of patients seen by the provider.

As noted earlier, the IRF-PPS simultaneously increased average reimbursement but decreased marginal reimbursement. Thus, the effects of the IRF-PPS on admission policy are a priori ambiguous. Furthermore, the effects of the IRF-PPS on profitability of patients might vary by patient characteristics and depend on how well the case mix adjustment of payments under the IRF-PPS matches the expected costs of treating patients. In general, IRFs have the incentive to increase admission probabilities for patients who became more profitable due to the implementation of the IRF-PPS. We refer to the change in the severity or type of patients seen at IRFs after the implementation of the IRF-PPS as the "selection"

\footnotetext{
${ }^{1}$ The supply of potential patients is determined by the decision of patients and hospital discharge planners who influence patient decision on whether and where to seek care. We assume that patient decisions to seek care at a particular provider are influenced by the intensity of care provided by the provider.
} 
effect of the IRF-PPS. Total capacity, measured either by number of IRFs or the number of Medicare certified beds, was increasing before and after the IRF-PPS (Huckfeldt et al. 2012). Also, occupancy rates were only 69\% in 2002 in the first year of the IRF-PPS (Medicare Payment Advisory Commission 2011b), so IRFs had capacity available to admit newly profitable patients after the IRF-PPS.

Now we consider the effect of changes in reimbursement on treatment policy or decisions about treatment intensity after a patient is admitted to a facility. The first order condition for treatment policy is:

$$
\left[c_{j}\right]: U_{c_{j}}=U_{\pi}\left(1-m_{j}-E(\pi)^{\partial N} / \partial c\right)
$$

The above condition shows that providers choose the treatment policy so that the marginal utility of an increase in treatment intensity for a particular patient equals the change in profit from increasing intensity times the marginal utility of profit. Changes in treatment intensity can affect profit through two channels. First, increases in treatment intensity increase the costs of treating existing patients and so reduce profit. Second, for-profit providers will choose treatment intensity to attract profitable patients subject to a capacity constraint (or a population constraint on profitable patients).

Equation (3) shows that treatment intensity and marginal reimbursement are positively related. An increase in marginal reimbursement reduces the loss in profit from increasing intensity and hence increases the intensity of treatment. An increase in average reimbursement increases the profits of the provider and hence reduces the marginal utility of profits. This in turn reduces the marginal value of loss in profits and hence increases the intensity of treatment. Increases in average reimbursement also increase the profitability of patients, thus making it more lucrative to attract patients by increasing intensity of treatment. Therefore, consistent with the results in Hodgkin and McGuire, our model also predicts that increases in average or marginal reimbursement will increase the intensity of care. Because the IRF-PPS reduced marginal reimbursement and increased average reimbursement, the effects of the IRF-PPS on intensity of treatment are ambiguous. It is important to note that our model and the model in Hodgkin and McGuire abstracts from the competitive environment facing providers. It is likely that the magnitude of the response by an IRF to the IRF-PPS will depend on the number of competitors for the IRF and the response of these competitors to the IRF-PPS. For example, the effect of the IRF-PPS on intensity of care depends on the elasticity of demand with respect to intensity of care and this elasticity likely depends on the competitive environment facing IRFs (Gaynor 2006).

Finally, the changes in treatment and admission policy of IRFs could also affect patient outcomes and have spillover effects on the behavior of other providers. For example, patients discharged "too early" from IRFs might be at higher risk of suffering adverse health outcomes, being readmitted to acute care hospitals or receiving care from other post-acute care providers.

\section{Data and Measures}

Our analysis examines post-acute care payments, costs, and health outcomes for patients discharged from acute care hospitals between January 2001 and June 2003 and whose principal diagnosis for the acute admission was stroke, lower extremity joint replacement, or hip fracture. These three conditions are the most prevalent conditions receiving care in IRFs, accounting for over 50\% of IRF cases in 2004 (Medicare Payment Advisory Commission 2006). Stroke patients are defined as those with a principal diagnosis in the acute hospital 
stay of intracerebral hemorrhage (diagnosis code 431.xx), occlusion and stenosis of precerebral arteries with infarction (433.x1), occlusion of cerebral arteries with infarction (434.x1), or acute but ill-defined cerebrovascular disease (436.xx). Hip fracture patients are defined as patients with a primary diagnosis of fractures of the neck of the femur (820.xx). Lower extremity joint replacement patients ${ }^{2}$ were defined as patients with a primary diagnosis for joint replacement, excluding hip fracture patients and patients with reattachment procedures. Hip fracture patients who were treated with partial or total joint replacement are included in the hip fracture group.

The unit of analysis is an episode of care consisting of an acute-care hospitalization for one of the three study conditions, which we call the index hospitalization, plus a fixed period of 90 days following discharge from the acute care hospital. We link a number of data sources to construct the outcome and explanatory variables used in our analysis. In the sections that follow, we describe the construction of the measures used in the analysis.

\subsection{Data}

4.1.1. Medicare Payments and Costs-We use the Medicare claims data linked to data from Medicare cost reports to construct the key Medicare payment and cost measures. The Medicare claims data include 100 percent Medicare standard analytic files (SAF) for home health agency claims for acute discharges occurring from January 2001 to June 2003, 100 percent Medicare standard analytic files (SAF) for skilled nursing facility claims for acute discharges occurring from January 2001 to June 2003, and 100 percent MEDPAR data for acute hospital, IRF, and long term care hospital claims following acute discharges from January 2001 to June 2003. We define IRF and other post-acute payment for each observation as total Medicare payments to IRFs (or other post-acute sites) occurring within a 90-day post-acute episode following an initial acute care discharge. Thus, if multiple IRF stays occur during a 90-day post-discharge period, they are counted as part of total IRF payments or costs for that episode. However, we do not count days (or admissions) that occur outside the 90-day post-discharge period as part of the episode. Such IRF stays form an additional episode (if preceded by an inpatient admission).

We calculate the costs per patient incurred by facilities using charges in the claims data and cost-to charge ratios from cost-reports. For hospital-based SNF, IRF, and LTCH costs, we multiply total charges accrued in a given facility by the facility's total calendar year cost-tocharge ratio. For stand-alone SNFs, we multiply routine costs-per-day by the total number of days, plus the ancillary cost-to-charge ratio by ancillary (departmental) charges. For HHAs, we multiply the number of visits by a facility's average calendar year cost per visit.

Our analysis relies on cost-to-charge ratios to deflate hospital charges into total costs of care. Thus, changes in accounting practices at the time of the PPS could lead to biased estimates. We do not know of any such changes during the sample period. Figure A.1 in the appendix plots average annual IRF cost-to-charge ratios and the fraction of cost-reports with missing cost-to-charge ratios from 1996 to 2008. Average IRF cost-to-charge ratios (the solid line) are falling over the sample period, but there is no obvious trend break at the time of the PPS in 2002. The fraction of IRFs missing cost-to-charge ratios increases by approximately two percentage points after the PPS, but this only represents around 20 additional facilities. Thus, there is little evidence of substantial changes in cost-to-charge reporting practices.

4.1.2. Health Outcomes-The main health outcome in the study is a binary measure of whether a patient died or was institutionalized in a nursing home at the end of the 90-day

\footnotetext{
${ }^{2}$ Referred to as "joint replacement" throughout this paper.
} 
period following discharge from the acute-care hospital as opposed to returning to the community. We identify deaths using Medicare denominator files, and whether patients were institutionalized using data from the Minimum Data Set, which reports information from all nursing homes that are certified by Medicare or Medicaid. These nursing homes, including those non-skilled nursing facilities that provide only custodial care, are required to regularly evaluate the health and functional status of patients who reside in the facility for longer than 14 days. We also measure readmissions to an acute-care hospital during the 90day period following discharge from the index hospitalization. ${ }^{3}$

4.1.3. Patient Characteristics-We use information from the index hospitalization and Medicare denominator files to measure patient characteristics ${ }^{4}$. For each patient we collect and control for the list of comorbidities developed by Elixhauser et al. (1998) ${ }^{56}$. We also measure a list of complications that occurred during the index hospitalization ${ }^{7}$ including post-operative pulmonary compromise; post-operative gastrointestinal hemorrhage; cellulitis or decubitus ulcer; septicemia; pneumonia; mechanical complications due to a device, implant, or graft; shock or arrest in the hospital; post-operative myocardial infarction; postoperative cardiac abnormalities other than AMI; procedure-related perforation or laceration; venous thrombosis and pulmonary embolism; acute renal failure; miscellaneous complications; delirium; and dementia. We control for hip fracture in stroke and joint replacement patients and for stroke in hip fracture and joint replacement patients. For hip fracture patients we include indicators for receiving a partial or total hip replacement, where the alternative treatments are internal fixation or no surgery. For stroke patients, we control for whether the stroke was hemorrhagic. For lower extremity joint replacement patients, we include indicators for hip versus knee replacement, whether a replacement was bilateral (i.e. both sides), whether the patient received multiple replacements, and whether a replacement was a revision of a prior replacement.

We use information from Medicare enrollment files to describe patient demographics including gender, age (controlled for separately in 5-year bands), race, and whether a patient was covered by Medicaid.

4.1.4. Acute-care Hospital Characteristics-Our analysis also controls for the characteristics of discharging acute-care hospitals, as these may influence post-acute care. We control for the Medicare patient percentage in the previous year, which we obtain from providers' cost reports to CMS. We use the Medicare Provider of Services file, a provider level database maintained by CMS, to determine a facility's ownership status (government,

\footnotetext{
${ }^{3}$ Longer post-acute episodes may capture later unrelated readmissions and subsequent costs, whereas shorter episodes may miss related costs, readmissions, and patient outcome. We examine the sensitivity of the results to differing post-acute episode lengths, in analysis not shown, and find similar results.

${ }^{4}$ Changes in reporting of patient characteristics post reform could affect our results, and diminish our ability to distinguish between effects driven by provider behavior versus patient composition. However, such reform-induced reporting changes would likely occur at IRFs. We obtain patient information from denominator files and the hospital claim preceding the IRF stay therefore such changes are unlikely to affect our results. In addition, we examine changes in the numbers of reported comorbidities and hospital complications over the sample period in section 4.1.3 and Figure A.2 and find smooth trends in numbers of comorbidities and complications with no discrete break at the time of payment reform.

${ }^{5}$ Comorbidities include AIDS, alcoholism, deficiency anemias, rheumatoid arthritis/ collagen vascular diseases, blood loss anemia, congestive heart failure, chronic pulmonary disease, coagulopathy, depression, diabetes without complications, diabetes with chronic complications, drug abuse, hypothyroidism, liver disease, lymphoma, fluid and electrolye disorders, metastatic cancer, other neurologic disorders, obesity, paralysis, peripheral vascular disease, psychoses, pulmonary circulation disease, renal failure, solid tumor without metastasis, peptic ulcer disease excluding bleeding, valvular disease, and weight loss. We do not include hypertension, as it is ubiquitous in these populations, or cardiac arrhythmias.

${ }^{6}$ We do not exclude or control for patients who are admitted to the hospital from a nursing home. As shown in Figure A.7., the fraction of patients among each of the sample conditions admitted from a nursing home stays relatively constant over the sample period. Moreover, the percentage of IRF admissions from nursing home residents is very low $(<2 \%)$.

${ }^{7}$ Because we are interested in the costs and health outcomes of post-acute-care, complications that occur during the index hospitalization are important measures of the severity of patients when they are discharged from the acute-care hospital.
} 
non-profit, or for-profit) and paired with information from the Area Resource File whether the hospital was in a rural area, an urban area, or adjacent to an urban area. We use information from the Acute Impact file to construct controls for wage index, average daily patient census, the severity of the facility's case-mix, teaching status (resident to average daily census ratio), the number of beds, and low-income patient percentage (disproportionate share patient percentage) as additional controls.

We exclude Medicare beneficiaries from Maryland and episodes initiating in Maryland hospitals (because Maryland hospitals are exempt from the PPS), patients that died during the index hospital stay, and episodes where Medicare was not the primary payer for the index hospital stay. In addition, we excluded patients who were in a Medicare HMO at any time during the month of their hospital discharge and the subsequent three months (identified using denominator files). Our analysis sample consists of 503,220, 776,249, and 645,213 episodes of care for hip fracture, joint replacement, and stroke respectively. We drop $1.7 \%, 2.8 \%$, and $1.8 \%$ of hip fracture, joint replacement, and stroke episodes respectively due to various missing data. Summary statistics for the analytic sample are presented in Table 1.

\section{Empirical Approach}

The goal of our empirical analyses is to understand the effects of the IRF-PPS on treatment and admission policies of IRFs for each of the three study conditions. To achieve this goal, we first describe changes in Medicare payments for IRF care. Next, we develop an empirical strategy that enables us to estimate the effect of the IRF-PPS on: (1) admission policies of IRFs, holding constant the composition of patients discharged from acute care hospitals and treatment, and (2) per-patient costs incurred by IRFs, holding constant the composition of patients receiving care in IRFs. Our strategy also enables us to estimate the extent to which changes in composition of patients discharged from acute-care or receiving care in IRFs affects study outcomes. We also investigate the effects of the IRF-PPS on average costs at other post-acute providers, acute readmissions, and mortality and institutionalization. For all analyses, we define the pre-PPS period as acute care discharges occurring during the four calendar quarters from January 2001 through December 2001 and the post-PPS period as the six quarters from 1 January 2002 through 30 June 2003. We estimate separate models for each study condition: hip fracture, lower extremity joint replacement, and stroke.

To summarize, our estimation approach involves empirically describing payment, admission, and treatment policies as a function of patients' observable characteristics for each study period or quarter. Next, we use these estimates to simulate outcomes in pre- and post-PPS periods for a constant patient cohort, to isolate the effects of the IRF-PPS on study outcomes. We also use these estimates to simulate study outcomes using a constant set of post-PPS parameters for pre and post-PPS patient cohorts, to isolate effects coming from changes in the composition of patients discharged from acute-care hospitals for admission probabilities and of patients treated in IRFs for payments and costs. This approach is similar in principle to the simulated instrumental variables strategy in Currie and Gruber (1996a, 1996b). We now describe our approach in detail.

We characterize the admission policy of IRFs by modeling the probability of using any IRF care for patients discharged from acute care hospitals with one of the study conditions. Specifically, we estimate a probit model with an indicator variable for using any IRF care during a 90-day post-acute episode as the dependent variable. The covariates in the model include patient demographics, comorbidities, complications, and characteristics of the discharging hospital, as described in the data section. We estimate separate probit models for each quarter. Thus, we allow the admission policy of IRFs or the probability of admissions 
to IRFs to change flexibly over time, including changes in the intercept that are common across all patients and changes in coefficients on covariates which produce differential changes in admission probabilities depending on patient characteristics or other covariates in the model.

$$
p_{i}^{q}=\Phi\left(\alpha^{q}+\beta^{q} X_{i}\right)
$$

Next, we use these estimated probit coefficients to estimate the probability of using IRF care in each quarter for a constant cohort of patients discharged from acute care in the first quarter of 2001. These estimates isolate the changes in admission policy of IRFs, as they project the probabilities of admission into IRFs in quarters 1 to 10 , with a constant sample of acute discharges from the first quarter of 2001. In order to estimate whether the IRF-PPS caused a change in the admission policies of the IRF we estimate the following interrupted time series model:

$$
p_{i}^{q}=\alpha+\delta_{0} \text { Quarter }+\sum_{\mathrm{k}=1 \text { to } 6} \delta_{\mathrm{k}} \text { Post }_{\mathrm{k}} \quad \text { (5.) }
$$

Where, $p_{i}^{q}$ is the probability of IRF admission for patient $i$ if he or she was discharged from acute care in quarter $q$. Quarter is a linear time trend in quarter of discharge and Post $t_{\mathrm{k}}$ is an indicator variable for the $\mathrm{k}^{\text {th }}$ quarter after implementation of IRF-PPS in January 2002. The coefficients of interest are $\delta_{\mathrm{k}}$ which show the average change in probability of admission to IRFs in the $\mathrm{k}^{\text {th }}$ quarter due to changes in admission policies of IRFs assuming the linear time trend in the four pre-PPS quarters continued.

We use a similar strategy to estimate the effects of the IRF-PPS on the treatment policies of IRFs. First, we estimate a series of OLS models for patients admitted to IRFs in each quarter that estimate the costs of IRF care as a function of patient demographics, comorbidities, complications, and hospital characteristics. Next, we use the estimated cost functions to calculate the costs of IRF care in each quarter for the cohort of patients that were admitted to IRFs in the first quarter of 2001. Finally, to determine whether the IRF-PPS caused a change in the treatment policies of the IRF we estimate the following interrupted time series model:

$$
c_{i}^{q}=\lambda+\lambda_{0} \text { Quarter }+\sum_{\mathrm{k}=1 \text { to } 6} \lambda_{\mathrm{k}} \text { Post }_{\mathrm{k}}
$$

The coefficients $\lambda_{1}$ to $\lambda_{6}$ describe the evolution of treatment policy or cost of care conditional on using an IRF following the implementation of the IRF-PPS.

We also simulate the effects of the IRF-PPS on payments in each quarter for a constant IRF patient cohort and estimate equation (6), in order to show the magnitude of changes in Medicare payments to providers.

We are also interested in estimating the "selection effects" of the IRF-PPS, that is, the extent to which payments and costs of care at IRFs changed due to changes in the types and severity of patients admitted (within each tracer condition), and the extent to which the probability of admission to an IRF changed as a result of changes in the composition of patients discharged from acute-care hospitals. To quantify these effects, we estimate the counterfactual costs for each cohort of patients admitted to IRFs from quarter 1 to quarter 10 using the cost function estimated for IRF patients in the second quarter of 2003, after the IRF-PPS phase-in was complete. Thus, we allow the patient population admitted to IRFs to change over time but keep the treatment policy fixed at 2003 levels. Next, we use the interrupted time series model described above to describe the evolution of selection effects 
following the implementation of the IRF-PPS. We conduct a similar analysis to estimate the extent to which the probability of admission into IRFs changed due to changes in the composition of patients being discharged from acute care hospitals. To quantify these effects, we estimate the counterfactual probability of IRF admission for each cohort of patients discharged from quarter 1 to quarter 10 using the admission function estimated in 2003 and use the interrupted time series model described above to describe the evolution of admission and selection effects following the implementation of the IRF-PPS.

Finally, we estimate the effects of the IRF-PPS on the costs of care for other post-acute care providers, the probability of hospital readmission, and the probability of death or institutionalization. The empirical strategy for these outcomes is similar to the strategy used for estimating treatment and admission policy effects. We omit a full description of the strategy in the interest of brevity. We also explore heterogeneous effects of the IRF-PPS by type of IRF (free standing versus hospital unit) and by patient characteristics.

The dependent variables in the main regressions are simulated values that are functions of the coefficient estimates from regressing Medicare payments, provider costs, or admissions on patients' observable characteristics in each quarter; these coefficients are themselves measured with error. Thus, conventional standard error estimates for the coefficient estimates in equations 5 and 6 may understate the true standard errors. Reflecting this, we estimate pairs clustered bootstrapped standard errors, as described in Cameron, Gelbach, and Miller (2008). ${ }^{8}$ Over 500 replications, we draw hospital referral region (HRRs, defined by Darmouth Medical School (1996)) with replacement from the original sample; simulate payments, costs, and admissions; and estimate $\lambda_{0}$ through $\lambda_{6}$ (and $\delta_{0}$ through $\delta_{6}$ ) from equations 5 and 6 . Then, the bootstrapped estimate of the standard error is just the standard deviation of each coefficient estimate over the 500 replications.

\section{Results}

We begin by describing our estimates of the IRF-PPS on Medicare payments to IRFs, resource use, and admissions. We then consider the spillover effects of the IRF-PPS on other post-acute settings and its effect on patient outcomes. Finally, we examine heterogeneous effects by patient characteristics and type of IRF.

\subsection{IRF payment, treatment, and admissions policies}

Using coefficient estimates from equations 5 and 6., panels a, c, and e of Figure 1 show Medicare payments to IRFs, IRF costs, and IRF admissions simulated for a fixed cohort of patients thus representing changes in payment, treatment, or admissions policies while panels $b, d$, and $f$ of Figure 1 simulate a fixed function over each subsequent cohort of patients and thus display changes in outcomes stemming from patient composition. In each case, the solid line represents the simulated outcome, the dotted line indicates the actual average in each quarter, and the dashed line represents the linear counterfactual trend had the PPS not been implemented (i.e., predictions where the indicator variables POST1POST6 are set to zero). Accompanying coefficient estimates are displayed in Table 2.

Figure 1a displays simulated and actual Medicare payments in each quarter from 2001 through the second quarter of 2003, for the cohort of hip fracture patients in IRFs discharged from acute care in the first quarter of 2001. The simulated payments represent changes in Medicare IRF expenditures stemming solely from changes in payment policy and imply a substantial increase in Medicare payments after PPS implementation relative to the payment trend prior to the PPS. The corresponding regression estimates in Table 2, column 1 show

\footnotetext{
${ }^{8}$ The method resamples from the clusters in the original sample with replacement.
} 
that by the end of the full phase-in in 2003, payments were nearly $\$ 2,400$ or more than 20 percent higher than pre-PPS levels. Actual average payments in Figure 1a are nearly identical to simulated payments, implying that most of the change in payments was primarily due to payment policy rather than changes in patient composition. Figure $1 \mathrm{~b}$ and Table 2, column 2 estimate changes in Medicare payments from patient composition more directly. Figure $1 \mathrm{~b}$ shows that simulated payments, predicted using a fixed payment function but allowing each cohort's composition to differ, are flat over the sample period. The corresponding coefficient estimates for POST1-POST6 in Table 2, column 2 are small and mostly statistically insignificant; however the coefficient on POST5 and POST6 are statistically significant but the magnitude of the estimates are small (about $1 \%$ of the average payment).

Next, we examine the effects of the dramatic increase in payments on IRF's treatment and admission policies. Figure 1c displays simulated costs, predicted over the sample period for a fixed cohort of IRF hip fracture patients. Despite Medicare's increased generosity, resource use for IRF patients initially decreased following the PPS before converging back towards the pre-existing time trend. The corresponding coefficient estimates for POST1POST6 in Table 2, column 3 show that costs were over $\$ 500$ dollars less per episode (an approximately 4 percent reduction) in the first quarter two quarters following the PPS, but these cost effects became smaller in magnitude and statistically insignificant by 2003 . The similarity of simulated and actual costs in Figure 1c implies that changes in costs were driven by treatment intensity conditional on patient characteristics rather than changes in patient composition. In addition, actual costs in each quarter track the pre-PPS trend relatively closely, supporting the linear specification for the pre-PPS time trend. Figure 1d and Table 2, column 4 confirm that changes in patient composition had little effect on provider costs, implying a minimal amount of selection within hip fracture patients admitted to IRFs.

Figure 1e displays actual IRF admission probabilities and simulated IRF admission probabilities for a fixed cohort of hospital discharges from 2001 quarter 1. Simulated admissions increased gradually immediately following the PPS. The coefficient estimates for POST5 and POST6 in Table 2, column 5 imply that admissions were over two percentage points higher by 2003. Figure 1f and Table 2, column 6 imply that the composition of hospital discharges changed over the sample period with later cohorts having a lower probability of being admitted to IRFs.

Tables 3 and 4 display the effects of the PPS on Medicare payments, provider costs, and admission probabilities for patients discharged from the hospital after a lower extremity joint replacement or stroke (respectively). In Table 3, Medicare payments increased by an even greater amount for joint replacement patients compared to hip fracture patients (29\% versus $20 \%$ ), but effects stemming from patient composition are mostly statistically insignificant. Reductions in provider costs after the PPS were similar to those for hip fracture patients $(\sim 4 \%)$ and were also temporary and not caused by changes in patient composition. Admission policy effects were smaller for joint patients, and the changing composition of hospital discharges increased (rather than decreased) the likelihood of IRF admission. Table 4 displays estimates for stroke patients and implies a similar pattern of results to hip fracture patients. Medicare payments increased substantially, driven primarily by payment policy, although patient composition shifted slightly in 2003 accounting for about a $1 \%$ increase in payments. Provider costs temporarily fell in the first year of the reform by a similar amount ( 4\%) and changes in patient composition had little effect on provider costs, implying little selection effects. Finally, changing admission policies for stroke patients increased admissions by almost two percentage points, but the changing 
composition of hospital discharges reduced admissions by nearly one percentage point by 2003.

In summary, our results imply that average Medicare payments per IRF patient increased after the IRF-PPS. This change in payment increased the probability of admission to IRFs for all three study conditions. However, we do find differential increases in admissions among the three tracer conditions. The greater increase in admissions among hip fracture patients relative to stroke and joint replacement patients may indicate that the profitability of hip fracture patients increased differentially and thus hip fracture patients were selectively admitted.

Despite increases in average Medicare payments per IRF patient, changes in treatment policy actually led to reduced resource use initially after the PPS, possibly reflecting decreased marginal payment. The average severity of patients seen in IRFs within each of the tracer conditions played little or no role in changing IRF payments or costs, but changes in the composition of patients discharged from acute hospitals after stroke and hip fracture led to some reduction in IRF admissions.

\subsection{Costs in other post-acute settings and patient outcomes}

Changes in admission and treatment policies in IRFs could in principle lead to changes in costs in other post-acute settings because at least for some patients the various settings are substitutes. For example, the increased admissions in IRFs could have diverted patients from other post-acute settings, while the decreased resource use could increase readmissions. In addition, changes in treatment patterns could affect health outcomes.

Figure 2 shows predicted average costs in other post-acute settings for hip fracture patients including home health agencies, skilled nursing facilities, and long-term care hospitals both together and separately. To compute simulated average costs across acute discharges, we first simulate admissions by applying each quarter's admission function for a specific other post-acute setting to a fixed cohort of patients discharged from acute care hospitals in the first quarter of 2001. Next, we simulate provider costs in each quarter by applying each quarter's cost function (estimated from patients seen in that post-acute setting) to a fixed cohort of patients discharged from hospitals in the first quarter of 2001. These simulated admissions and cost measures are then multiplied to provide simulated unconditional costs for each quarter. In this calculation, the simulated unconditional costs only change with changes in admissions and treatment policies of other PAC settings, because the patient samples are held constant. In each case, the solid line represents average simulated unconditional costs in each quarter, the dashed line represents the counterfactual trend without the PPS (i.e. POST1 through POST6 in equation 5 set to zero), and the dotted line represents actual average unconditional costs in each quarter.

Figure 2 shows little change in total post-acute costs across other settings or for specific settings after the IRF-PPS for hip fracture patients, with the exception of a small reduction in SNF costs starting in 2003, possibly reflecting decreased average payments after the expiration of add-on payments at the end of fiscal year 2002 (Medicare Payment Advisory Commission 2007). Table 5 shows estimated changes in other post-acute costs across settings after the IRF-PPS for each condition. The results in column 1 imply little change in other post-acute costs for hip fracture patients in the first four quarters following the IRF PPS, with a small decrease in 2003. In contrast, column 2 shows an increase in other postacute costs for joint patients in the first four quarters after the PPS that diminishes and becomes statistically insignificant in 2003. Column 3 shows mostly statistically insignificant reductions in other post-acute costs for stroke patients in the first four quarters after the PPS 
that become more negative and statistically significant in 2003 (representing an approximately 10 percent decrease relative to the overall average).

Table 6 displays results for each separate post-acute setting by condition. There is little persistent or statistically significant change in home health agency use for hip fracture (column 1) or stroke patients (column 3), but column 2 shows a statistically significant increase in home health costs by joint replacement patients of up to $\$ 101$ (relative to a mean of $\$ 1081$ ). The second panel shows reductions in skilled nursing facility use by 2003 for hip fracture and stroke patients, but as described above, these reductions may have been due to reductions in Medicare payments to skilled nursing facilities rather than the IRF PPS. In contrast, skilled nursing facility costs increased for joint replacement patients in 2002, but diminished and became statistically insignificant by 2003 (i.e. POST5 and POST6). However, this increase and decrease could also have been driven by the implementation and subsequent expiration of skilled nursing facility "add-on" payments, described above. Columns 7 and 8 show no significant change in long term care hospital use for hip fracture or joint replacement patients. However, column 9 shows large reductions in long term care hospital costs for stroke patients after the IRF-PPS (relative to the positive and statistically significant pre-reform trend), suggesting that some new IRF patients may have previously gone to long term care hospitals. However, a prospective payment system for long term care hospitals was implemented in October 2002, which may be responsible for much of this decrease.

In summary, these results suggest that there may have been substitution towards home health agencies and skilled nursing facilities for joint replacement patients, but the delayed timing of changes in other post-acute costs for hip fracture and stroke suggest that they may be driven by other Medicare payment changes. Thus, overall we find little evidence of substitution across post-acute settings for these conditions.

Table 7 displays estimated changes in hospital readmissions and whether patients were institutionalized or dead at the end of the 90-day post-discharge episode. We followed our previous method of simulating outcomes by first estimating "predicted outcome" functions for each quarter in our sample as a function of observable characteristics, and then using those estimated equations to simulate outcomes for a fixed patient cohort (in this case acute discharges in the first quarter of 2001). Reflecting seasonal patterns in readmissions and mortality, the regression model includes a "POST-PPS" indicator interacted with calendar quarter, calendar quarter main effects, and a linear time trend. Table 7, columns 1-3 show statistically insignificant changes in readmission rates for hip fracture or stroke patients; however there is some evidence of an increase in readmission rates for joint replacement patients (though insignificant for the fourth quarter). The results in columns 4-6 imply mixed effects of the IRF-PPS on the fraction of patients who are dead or institutionalized. Column 4 shows mostly statistically insignificant changes of mixed direction for hip fracture patients. Column 5 shows a roughly 10 percent increase in mortality or institutionalization for joint replacement patients. However, column 6 shows no statistically significant change for stroke patients. Overall, these estimates suggest no change in patient health outcomes after the IRF-PPS for hip fracture and stroke patients.

\subsection{Stratified estimates for freestanding and hospital-based rehabilitation facilities}

Inpatient rehabilitation facilities are operated either as departments within acute hospitals or as separate freestanding facilities. At the time of the IRF-PPS, 973 IRFs were hospital-based and 215 were freestanding (Medicare Payment Advisory Commission 2006). However, the fraction of IRF patients seen in freestanding facilities is disproportionate to the number of facilities because of their greater size. 
We may expect differing responses to the IRF-PPS by freestanding and hospital-based facilities based on the experiences in other post-acute settings. White (2003) finds that freestanding skilled nursing facilities were more responsive to the new payment incentives in the Skilled Nursing Facility Prospective Payment System than hospital-based facilities and speculates that being part of a larger acute hospital may reduce pressure to maximize revenue under a new payment system. We explore the presence of different patterns in Figure 3, which displays simulated payments and costs for a fixed cohort of IRF patients discharged from the hospital in the first quarter of 2001, estimated and simulated separately for patients in freestanding and hospital-based facilities. Figures $3 a$ and $b$ show that Medicare payments to freestanding IRFs for hip fracture patients were slightly higher than freestanding facilities and increased a comparable amount after the IRF-PPS. However, Figures $3 \mathrm{c}$ and $\mathrm{d}$ show that freestanding IRFs reduced resource use dramatically after the IRF-PPS relative to hospital-based IRFs. Figure 3e shows that the probability of admission to a freestanding or hospital-based IRF changed little immediately following the PPS, but the probability of admission to a hospital-based facility eventually increased and accounted for much of the increase in admission for hip fracture patients.

Table 8 displays the coefficient estimates underlying Figure 3. Columns 1 and 2 show that freestanding and hospital-based IRFs experienced similar increases in Medicare payment for hip fracture patients after the IRF-PPS, with slightly larger increases in hospital-based facilities by 2003. However, columns 3 and 4 show that freestanding facilities reduced resource use by 1518 dollars (or over 10 percent) for hip fracture patients immediately following the IRF-PPS while initial reductions in resource use in hospital-based facilities were considerably smaller and statistically insignificant. Figures A5 and A6 and Tables A1 and A2 (in the Appendix) show similar patterns for joint replacement and stroke patients.

In summary, these results imply that, as in the SNF PPS, freestanding facilities were more likely to respond to the new incentive (specifically reductions in marginal payment) to reduce resource use in order to maximize profit margin. However, as in the non-stratified results, these effects were temporary.

\subsection{Heterogeneous effects across patient characteristics}

Finally, we investigate whether the effects of the IRF-PPS on provider behavior differ by patient characteristics. Case mix groups - the basis of the IRF prospective payment system are not only defined based on a patient's primary diagnosis requiring rehabilitation, but also by cognitive and motor function and explicitly by age for some diagnoses. In addition, casemix group (and thus payment) also depends on the presence of comorbidities. Depending on the level of historical payments under the prior cost-based system, certain case mix groups may have received a greater increase in payment (relative to the average historical payment) than others, and this may lead to heterogeneity in the effects of the PPS on costs and admissions. In addition, the level of provider discretion to change admission probabilities and treatment patterns might also vary by patient characteristics. For example, it might be more difficult to deny admission to a patient with poor functional status or providers might find it easier to reduce the intensity of care for less severely ill patients.

To investigate differential provider response by patient characteristics we simulate IRF payments and costs for a constant cohort of IRF patients (from 2001 q1) and IRF admissions for a constant cohort of hospital discharges (also from $2001 \mathrm{q} 1$ ) in the first quarter of the sample (2001 q1) and the last quarter of the sample (2003 q2). We examine separate effects by age groups (age $65-74,75-84$, and $85+$ ), the presence of comorbidities $(0,1$, and $2+$ ), and number of complications from the acute care hospital stay $(0$, and $1+)$ for each condition. We calculate bootstrapped standard errors and test for significant differences between groups using chi-square tests. Results are displayed in Table 9. 
The first three rows of Table 9 show that IRFs received a greater increase in payments (\# sim pay) for patients aged 65-74 than older patients (ages 75-84 or ages 85+) for patients with hip fracture or joint replacement. For stroke patients, the middle age category (75-84) received the largest increase in payment after the PPS. In each case, differences in payments across age categories were statistically significant across groups. Among hip fracture patients, we do not reject that changes in costs or admissions were the same across the three age groups. Among joint replacement patients, the youngest patients received the largest increase in costs but differences in changes in admissions are statistically insignificant. Stroke patients ages 75-84 represent the largest increase in payment for providers, but differences in changes in costs and admissions were also statistically insignificant across age groups.

Next, we examine heterogeneity by the level of comorbidity. Hip fracture patients with one or two or more comorbidities represented a smaller increase in payments than patients with no comorbidities (although we do not reject that the three groups exhibited equivalent changes in payment); they also received a smaller increase in admissions (changes in costs are also statistically insignificant). There is no statistically significant difference in changes in payments, costs, or simulated admissions across level of comorbidity among joint replacement patients. Stroke patients with two or more comorbidities represented the largest increase in payment; however, we find that differences in costs and admissions were statistically insignificant across comorbidity categories. We find similarly mixed and mostly insignificant differences across conditions when considering heterogeneity in the occurrence of hospital complications.

In summary, we observe some heterogeneity in payment changes within a diagnosis across patient characteristics, such as age, level of comorbidity, and with the presence of hospital complications. In some cases, within-condition age or comorbidity groups receiving larger payment increases also experience larger increases in provider costs or IRF admissions, but in other cases larger payment increases are associated with smaller increases in costs or admissions. As noted earlier, this might reflect differing degrees of provider discretion to change admission probabilities and treatment patterns by patient characteristics.

\section{Conclusion}

In this paper we investigated the impacts of the IRF-PPS. We developed a conceptual framework showing that the PPS may affect facilities' admission criteria, as well as the intensity with which IRFs treat patients. We then employed an empirical strategy that isolated changes in resource use due to changes in treatment policy and patient selection, and decomposed changes in admission probabilities into changes in admission policies and shifts in the composition of patients discharged from acute care hospitals.

We find that average Medicare IRF expenditures across all acute discharges for hip fracture, lower extremity joint replacement, and stroke increased substantially after the IRF-PPS. The increases were driven by increased payment for the types of patients already seen in IRFs and through increased IRF admissions. Despite increased payment, treatment intensity in IRFs decreased immediately after the PPS, implying that reduced marginal reimbursement dominated the increased average reimbursement. The composition of patients within diagnostic groups seen in IRFs changed little over this period, reflecting a minimal amount of selection or "cream skimming" by providers after the PPS; however, differential increases in admissions across diagnostic groups implies potential condition-level selection. Average costs for other post-acute providers increased immediately after the PPS for joint replacement patients, suggesting that additional rehabilitation occurred elsewhere after the PPS. However, we find less evidence for changes in other post-acute use that are attributable 
to the IRF-PPS for hip fracture and stroke patients. We find little impact of the PPS on acute readmissions or mortality and institutionalization, except for joint replacement patients, who exhibited significant increases in these outcomes after the PPS, potentially due to reductions in IRF treatment intensity.

The findings of this study should be viewed in light of its limitations. Our estimates of the effects of the IRF-PPS on costs and outcomes might be biased due to changes in patient characteristics (such as changes in functional status) that are coincident with the implementation of the IRF-PPS but not evident in the claims data that we use for our analysis. However, the likelihood of such bias is small given that we find little or no change in observable characteristics of patients and others studies using richer clinical data and measures of functional status find similar results(Paddock et al. 2007). However, it is important to note that we compare average patient characteristics before and after the IRFPPS. It is possible that marginal patients who were admitted to IRFs as a result of the PPS were different, but that these differences are not apparent in the average characteristics of patients.

Our findings of the effects of the IRF-PPS on other post-acute care providers might be biased by payment or other changes at these post-acute care settings that were coincident with the implementation of the IRF-PPS. We mitigate this bias by looking at a narrow window of time before and after the IRF-PPS where no major payment changes occurred in other post-acute settings. However, this limits our ability to analyze the long term effects of the IRF-PPS.

Finally, we find little evidence that the IRF-PPS affected rates of readmission, mortality and institutionalization for two out of three conditions analyzed. However, we cannot rule out the possibility that the IRF-PPS affected quality of life or functional status of patients with these conditions.

Policymakers continue to wrestle with the joint goals of reducing the growth rate of Medicare expenditures while improving quality of care and minimizing acute readmissions. Policy prescriptions range from tweaking post-acute payment updates to proposals for a single "bundled" payment for a combined acute plus post-acute episode, possibly including physician cost. Our results show that such policies can have important effects on both the likelihood of receiving post-acute care as well as the intensity of post-acute care. The results also suggest that these changes in use of care at both the extensive and intensive margin might not translate in worse patient outcomes; that is, payment reform can in principle achieve the goal of reducing health care costs without adversely affecting patient outcomes. The extent to which current or future reform proposals will achieve the elusive goal of reducing costs without adversely affecting patient outcomes is an open question. The effects will depend on the magnitude of changes in marginal and average reimbursement, the extent to which these changes are sustained in the long run, and whether the reforms focus on a particular site of care or all providers.

\section{Supplementary Material}

Refer to Web version on PubMed Central for supplementary material.

\section{Acknowledgments}

The authors wish to acknowledge the role of Melinda Beeuwkes Buntin who was a critical part of the research team at the outset of this project. This study was funded in part by National Institute of Aging (NIA) Grant R01AG031260 and AHRQ Grant R01-HS018541. 


\section{References}

Buntin MB, Carter GC, Hayden O, Hoverman C, Paddock SM, Wynn BO. Inpatient rehabilitation use before and after implementation of the IRF prospective payment system. RAND Technical Report 257. 2006

Buntin MB, Colla CH, Deb P, Sood N, Escarce JJ. Medical Spending and Outcomes After Postacute Care for Stroke and Hip Fracture. Medical Care. 2010; 48(9):776-84. [PubMed: 20706167]

Cameron AC, Gelbach JB, Miller DL. Bootstrapped improvements for inference with clustered errors. The Review of Economics and Statistics. 2008; 90(3):414-27.

Currie J, Gruber J. Health Insurance Eligibility, Utilization of Medical Care, and Child Health. Quarterly Journal of Economics. 1996a; 111(2):431-66.

Currie J, Gruber J. Saving Babies: The Efficacy and Cost of Recent Changes in the Medicaid Eligibility of Pregnant Women. Journal of Political Economy. 1996b; 104(6):1263-96.

Cutler DM. The Incidence of Adverse Medical Outcomes Under Prospective Payment. Econometrica: Journal of the Econometric Society. 1995:29-50.

Dartmouth Medical School. The Dartmouth Atlas of Health Care. Chicago: AHA Press; 1996.

Elixhauser A, Steiner C, Harris DR, Coffey RM. Comorbidity measures for use with administrative data. Medical Care. 1998; 36(1):8-27. [PubMed: 9431328]

Ellis RP, McGuire TG. Hospital response to prospective payment: moral hazard, selection, and practice style effects. Journal of Health Economics. 1996; 15:257-77. [PubMed: 10159442]

Gaynor M. What do we know about competition and quality in health care markets? National Bureau of Economic Research. 2006

Hodgkin D, McGuire TG. Payment levels and hospital response to prospective payment. Journal of Health Economics. 1994; 13:1-30. [PubMed: 10134436]

Huckfeldt P, Sood N, Romley J, Malchiodi A, Escarce J. Medicare Payment Reform and Provider Entry and Exit. Mimeo. 2012

Kahn KL, Keeler EB, Sherwood MJ, Rogers WH, Draper D, Bentow SS, Reinisch EJ, Rubenstein LV, Kosecoff J, Brook RH. Comparing outcomes of care before and after implementation of the DRGbased prospective payment system. JAMA. 1990; 264(15):1984-8. [PubMed: 2120477]

McCall N, Korb J, Petersons A, Moore S. Reforming Medicare payment: early effects of the 1997 Balanced Budget Act on post-acute care. The Milbank Quarterly. 2003a; 81(2):277-303. [PubMed: 12841051]

McCall N, Petersons A, Moore S, Korb J. Utilization of home health services before and after the Balanced Budget Act of 1997: what were the initial effects? Health Services Research. 2003b; 38(1):85-106. [PubMed: 12650382]

McKnight R. Home care reimbursement, long term care utilization, and health outcomes. Journal of Public Economics. 2006; 90:293-323.

Medicare Payment Advisory Commission. Report to the Congress: Medicare Payment Policy. Washington D.C.: Medpac; 2006. Inpatient rehabilitation facility services.

Medicare Payment Advisory Commission. Report to the Congress: Medicare Payment Policy. Washington D.C.: Medpac; 2007. Skilled nursing facility services.

Medicare Payment Advisory Commission. Payment Basics. Washington D.C.: Medpac; 2011a. Inpatient Rehabilitation Facilities Payment System.

Medicare Payment Advisory Commission. Report to the Congress: Medicare Payment Policy. Medpac; 2011b. Inpatient rehabilitation facility services: Assessing payment adequacy and updating payments.

Medicare Payment Advisory Commission. A Data Book: Health care spending and the Medicare program. Washington D.C.: Medpac; 2012. Post-acute care.

Newhouse, JP. Pricing the priceless: a health care conundrum. Cambridge (MA): The MIT Press; 2002.

Newhouse JP, Byrne DJ. Did Medicare's Prospective Payment System Cause Length of Stay to Fall? Journal of Health Economics. 1988; 7(4):413-6. [PubMed: 10303151] 
Paddock SM, Escarce JJ, Hayden O, Buntin MB. Did the medicare inpatient rehabilitation facility prospective payment system result in changes in relative patient severity and relative resource use? Medical Care. 2007; 45(2):123-30. [PubMed: 17224774]

Rogers WH, Draper DD, Kahn KL, Keeler EB, Rubinstein LV, Kosecoff J, Brook KH. Quality of care before and after implementation of the DRG-based prospective payment system. JAMA. 1990; 264(15):1989-94. [PubMed: 2120478]

Sood N, Buntin MB, Escarce JJ. Does How Much and How You Pay Matter? Evidence from the Inpatient Rehabilitation Care Prospective Payment System. Journal of Health Economics. 2008; 27(4):1046-59. [PubMed: 18423657]

Stineman MG. Prospective payment, prospective challenge. Archives of Physical Medicine and Rehabilitation. 2002; 83:1802-05. [PubMed: 12474191]

White C. Rehabilitation Therapy In Skilled Nursing Facilities: Effects of Medicare's New Prospective Payment System. Health Affairs. 2003; 22(3):214-23. [PubMed: 12757287] 
(a) Payments simulated for fixed cohort of IRF patients (payment policy effects)

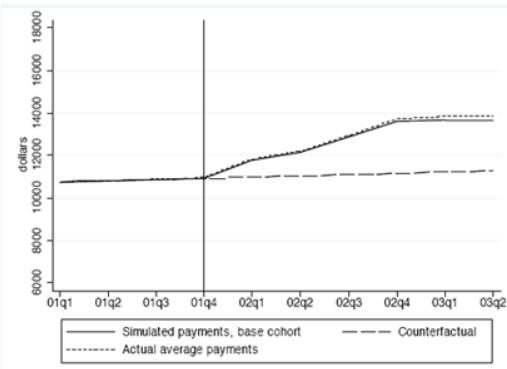

(c) Costs simulated for fixed cohort of IRF patients (treatment policy effects)

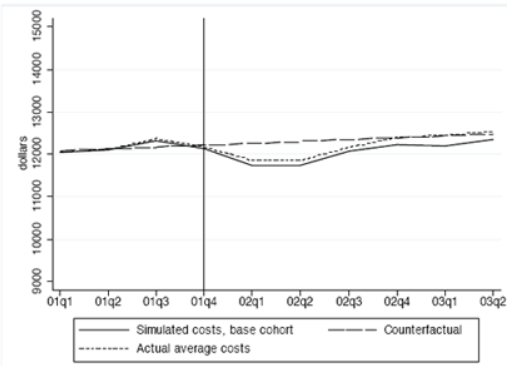

(e) Admissions simulated for fixed cohort of hospital discharges (admission policy effects)

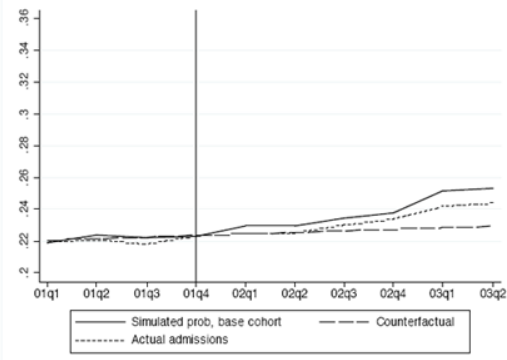

(b) Fixed payment function simulated for each IRF cohort (selection effects)

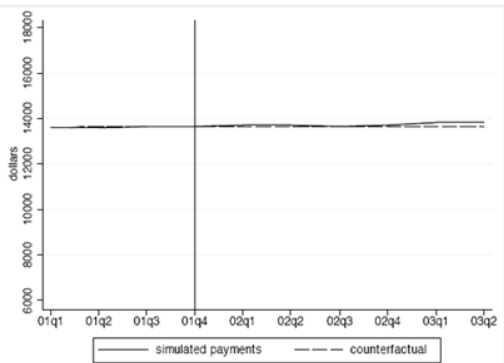

(d) Fixed cost function simulated for each IRF cohort (selection effects)

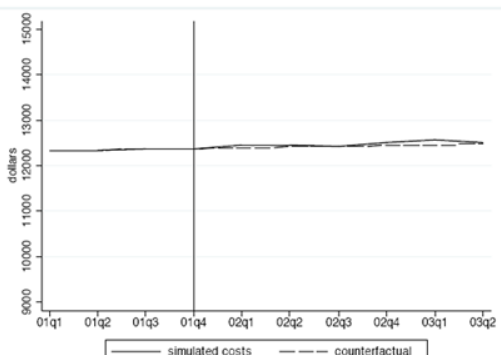

(f) Fixed admission function simulated for each hospital discharge cohort (composition effects)

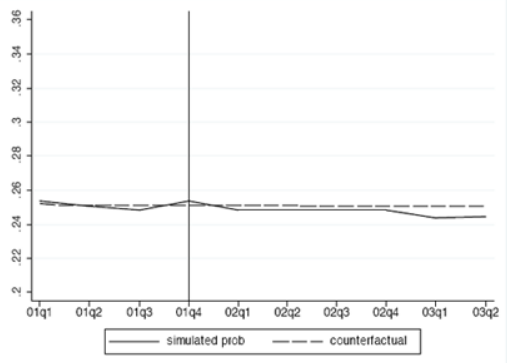

Figure 1.

Inpatient rehabilitation facility (IRF) payments, costs, and admissions for patients discharged from hospital after hip fracture

Note: Solid lines show average simulated payments, costs, or admissions; dashed lines show predicted values from regressions setting POST1-POST6=0; and dotted lines show actual averages. Vertical line indicates quarter before Medicare IRF-PPS. 
(a) Other post-acute costs (home health+ skilled nursing+ long term care hospital)

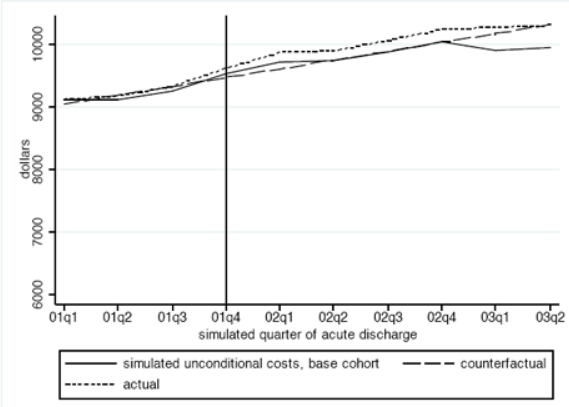

(c) Skilled nursing costs

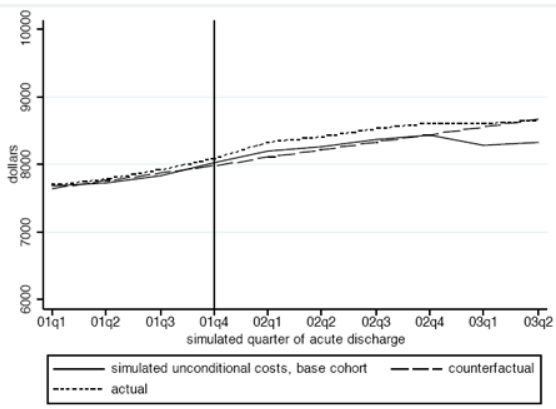

(b) Home health costs

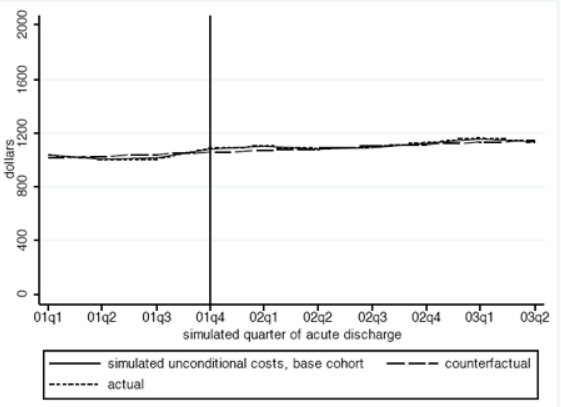

(d) Long-term care hospital costs

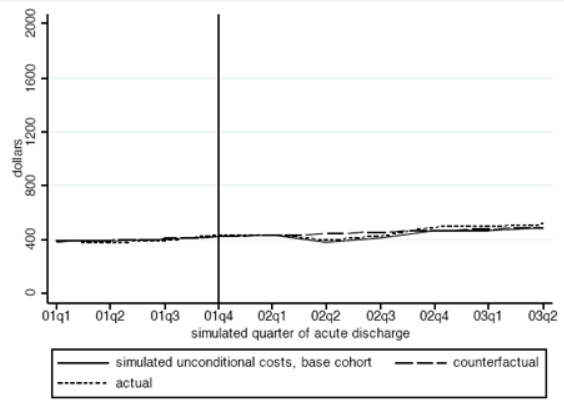

Figure 2.

Provider costs in other post-acute settings, hip fracture patients

Note: Solid lines show unconditional costs in other post-acute settings simulated for a fixed cohort of hospital discharges, dashed lines show predicted values holding POST1-POST6=0, and dotted lines indicates actual unconditional costs in each quarter. Vertical line indicates quarter before Medicare IRF Prospective Payment System (IRF-PPS). 
(a) Medicare payments to freestanding IRFs

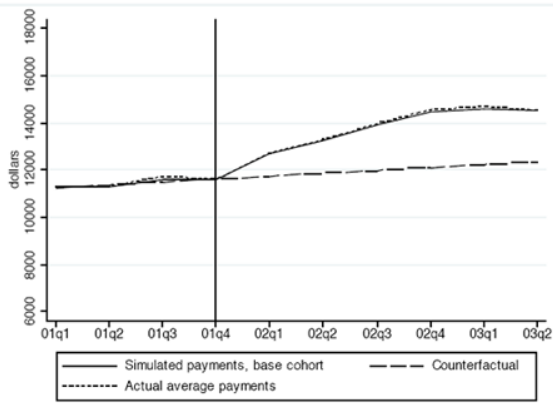

(c) IRF costs, freestanding IRFs

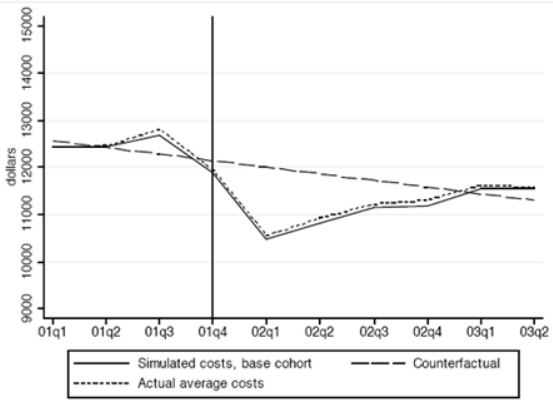

(e) Probability of hospital-based and freestanding IRF admission

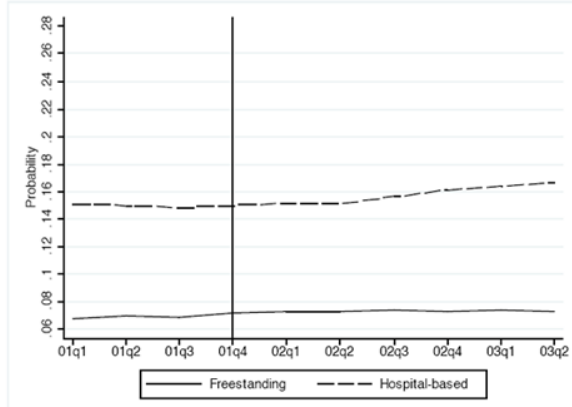

(b) Medicare payments to hospital-based IRFs

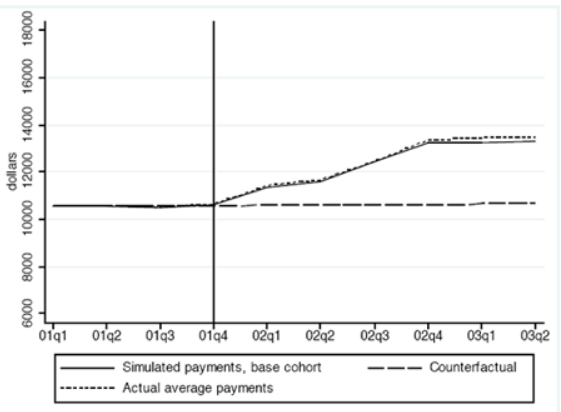

(d) IRF costs, hospital-based IRFs

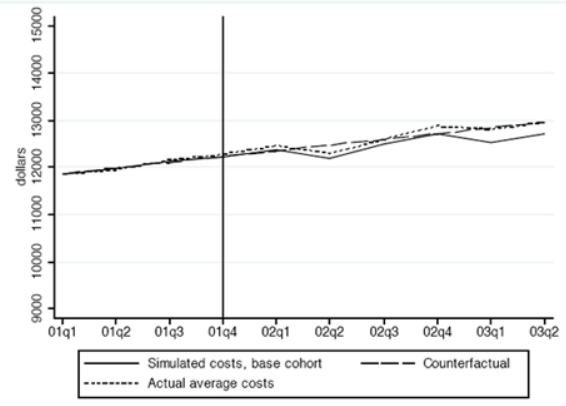

Figure 3.

IRF payments and costs simulated for fixed patient cohorts in freestanding and hospitalbased IRFs, hip fracture patients

Note: Solid lines show average of simulated values in each quarter, dashed line shows predicted values holding POST1-POST6=0, and dotted line shows average of actual values. Vertical line indicates quarter before Medicare IRF Prospective Payment System (IRF PPS). 


\section{Table 1}

Summary statistics

\begin{tabular}{|c|c|c|c|}
\hline & $\begin{array}{c}\text { (1) } \\
\text { Hip fracture }\end{array}$ & $\begin{array}{c}(2) \\
\text { Joint replacement }\end{array}$ & $\begin{array}{c}(3) \\
\text { Stroke }\end{array}$ \\
\hline Age & 82 & 73 & 78 \\
\hline Male & 0.24 & 0.35 & 0.41 \\
\hline White & 0.93 & 0.91 & 0.82 \\
\hline Urban & 0.72 & 0.70 & 0.72 \\
\hline Adjacent & 0.16 & 0.16 & 0.16 \\
\hline Rural & 0.13 & 0.14 & 0.13 \\
\hline Any comorbidities & 0.82 & 0.58 & 0.75 \\
\hline Total comorbidities & 1.64 & 0.93 & 1.34 \\
\hline Any complications & 0.36 & 0.08 & 0.27 \\
\hline Total complications & 0.43 & 0.10 & 0.33 \\
\hline Any IRF & 0.23 & 0.34 & 0.22 \\
\hline IRF payments & 2,788 & 2,807 & 3,573 \\
\hline Any other post-acute & 0.88 & 0.71 & 0.62 \\
\hline Post-acute payments & 9,303 & 3,373 & 5,692 \\
\hline Any readmission & 0.26 & 0.13 & 0.28 \\
\hline Readmission payments & 2,495 & 1,198 & 2,745 \\
\hline Institutionalized or mortality at 90 days & 0.38 & 0.03 & 0.33 \\
\hline $\mathrm{N}$ & 494,742 & 754,399 & 633,879 \\
\hline
\end{tabular}

Notes: Table displays summary statistics for 90-day episodes following acute discharges for hip fracture, joint replacement, and stroke occurring from January 2001 through June 2003. 


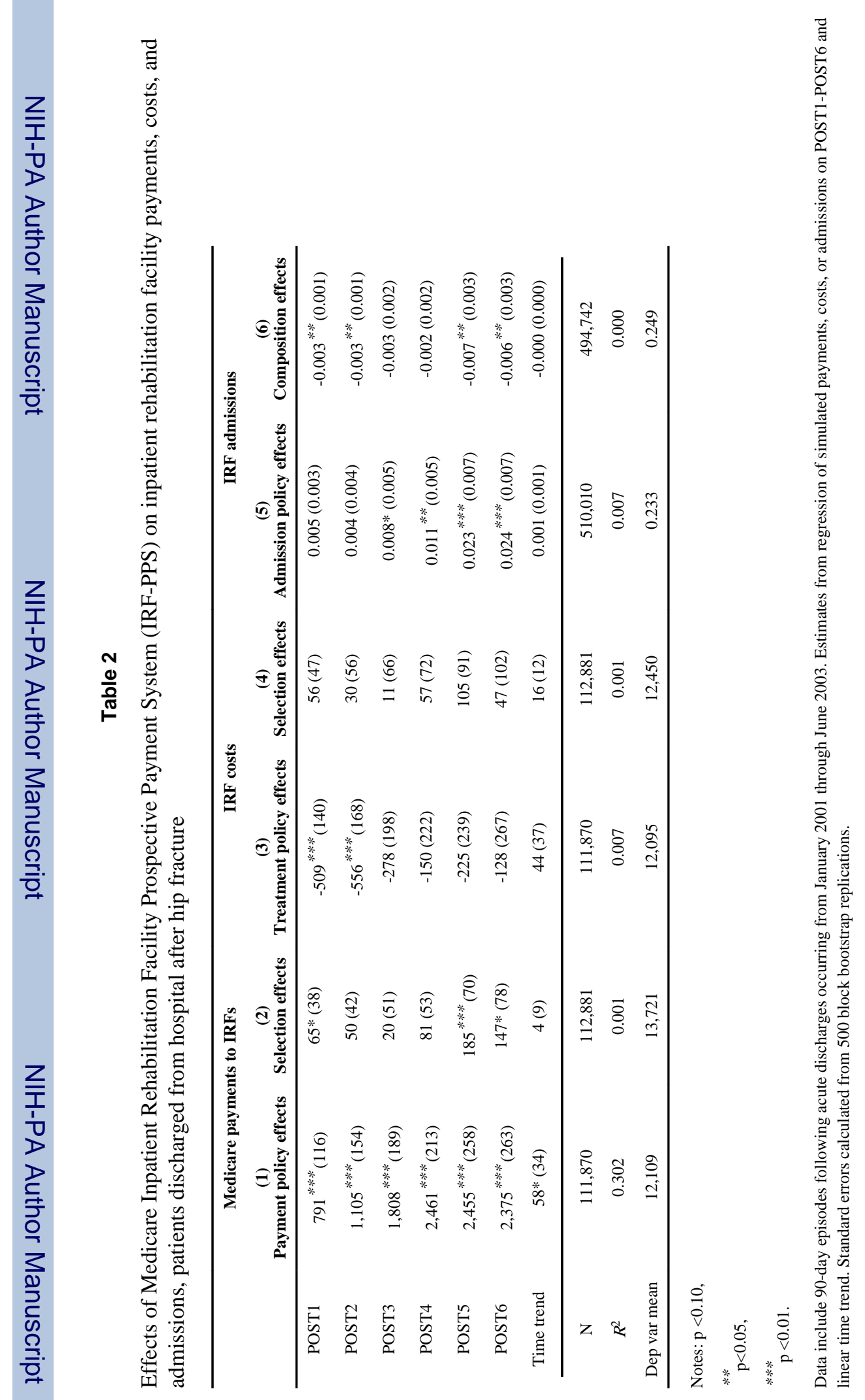

$J$ Health Econ. Author manuscript; available in PMC 2014 September 01. 


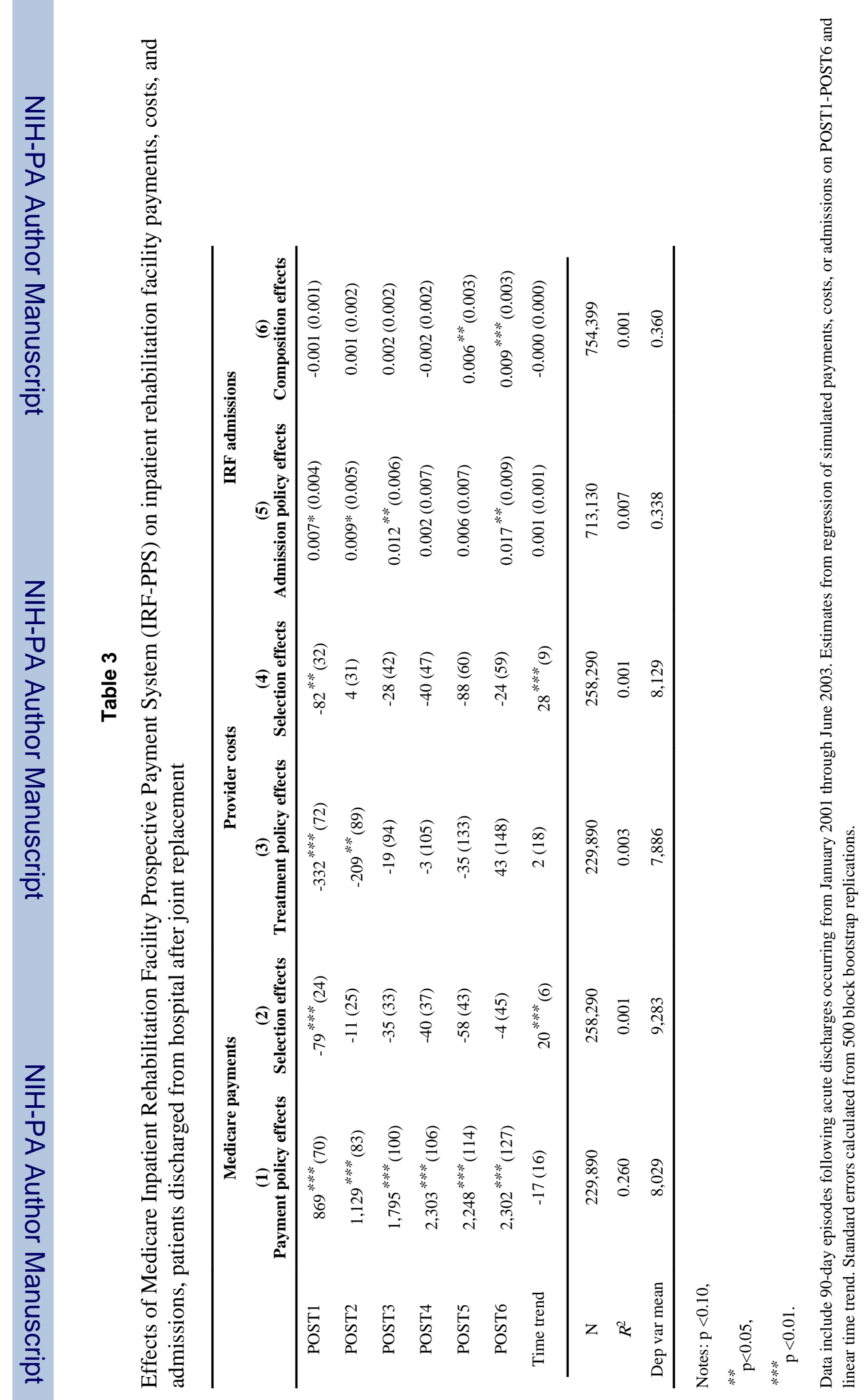




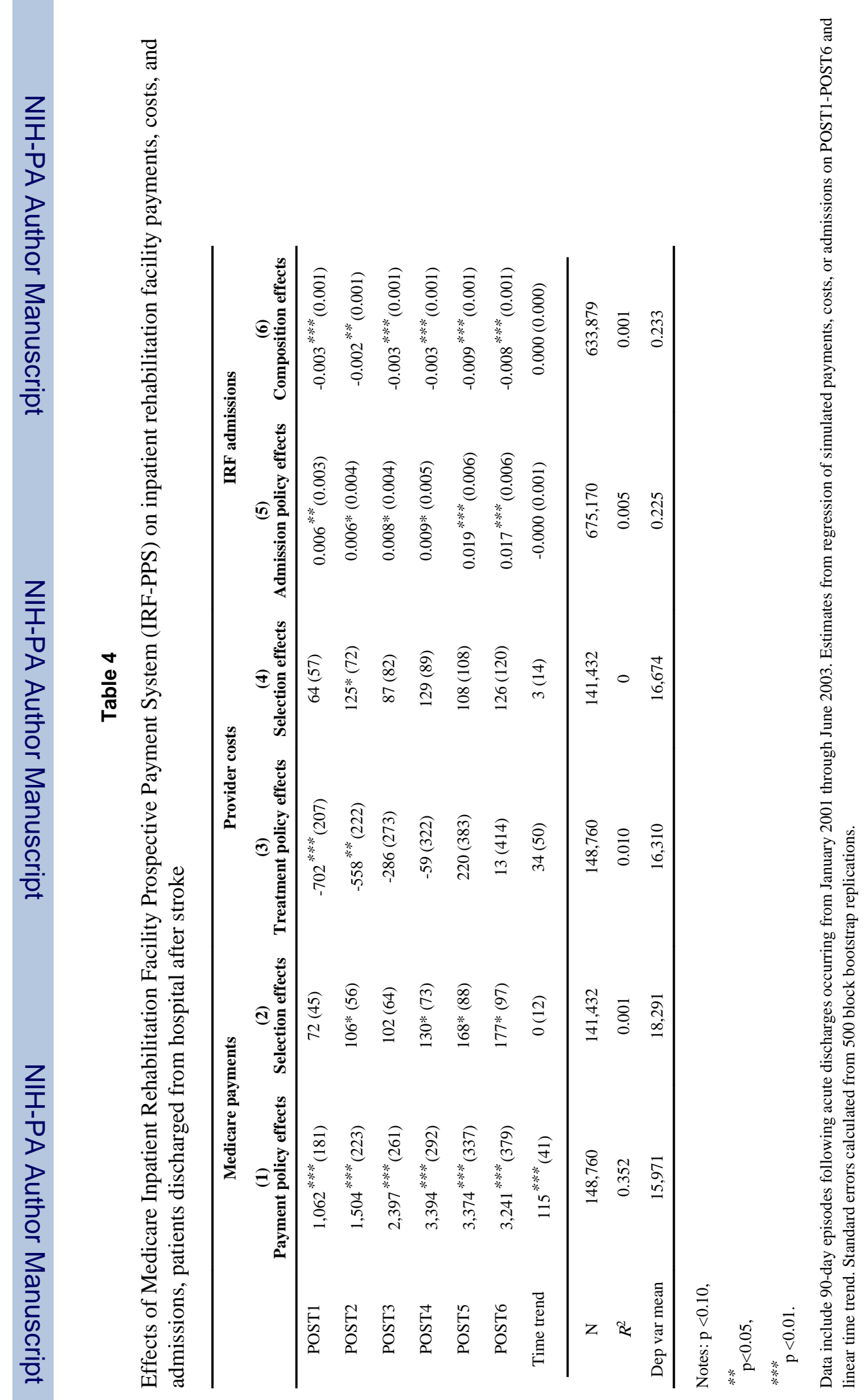


Table 5

Spillover effects of IRF-PPS on unconditional costs in other post-acute settings (skilled nursing facilities +home health agencies+long term care hospitals)

\begin{tabular}{lccc}
\hline & $\begin{array}{c}(\mathbf{1}) \\
\text { Hip fracture }\end{array}$ & $\begin{array}{c}(\mathbf{2}) \\
\text { Joint replacement }\end{array}$ & $\begin{array}{c}(\mathbf{3}) \\
\text { Stroke }\end{array}$ \\
\hline POST1 & $111(98)$ & $158^{* * *}(33)$ & $-63(73)$ \\
POST2 & $-18(105)$ & $179^{* * *}(42)$ & $-134(90)$ \\
POST3 & $-20(115)$ & $179^{* * *}(51)$ & $-166^{*}(100)$ \\
POST4 & $4(142)$ & $170^{* * *}(58)$ & $-150(126)$ \\
POST5 & $-268(171)$ & $94(70)$ & $-350^{* *}(145)$ \\
POST6 & $-352^{*}(183)$ & $58(89)$ & $-617^{* * *}(164)$ \\
Time trend & $140^{* * *}(23)$ & $-9(10)$ & $107^{* * *}(20)$ \\
\hline N & 509,939 & 713,130 & 675,095 \\
$R^{2}$ & 0.022 & 0.001 & 0.004 \\
Dep var mean & 9,622 & 3,181 & 6,026 \\
\hline
\end{tabular}

Notes: $\mathrm{p}<0.10$,

* $<<0.05$

**** $\mathrm{p}<0.01$

Data include 90-day episodes following acute discharges occurring from January 2001 through June 2003. Estimates from regression of simulated unconditional costs on POST1-POST6 and linear time trend. Standard errors calculated from 500 block bootstrap replications. 


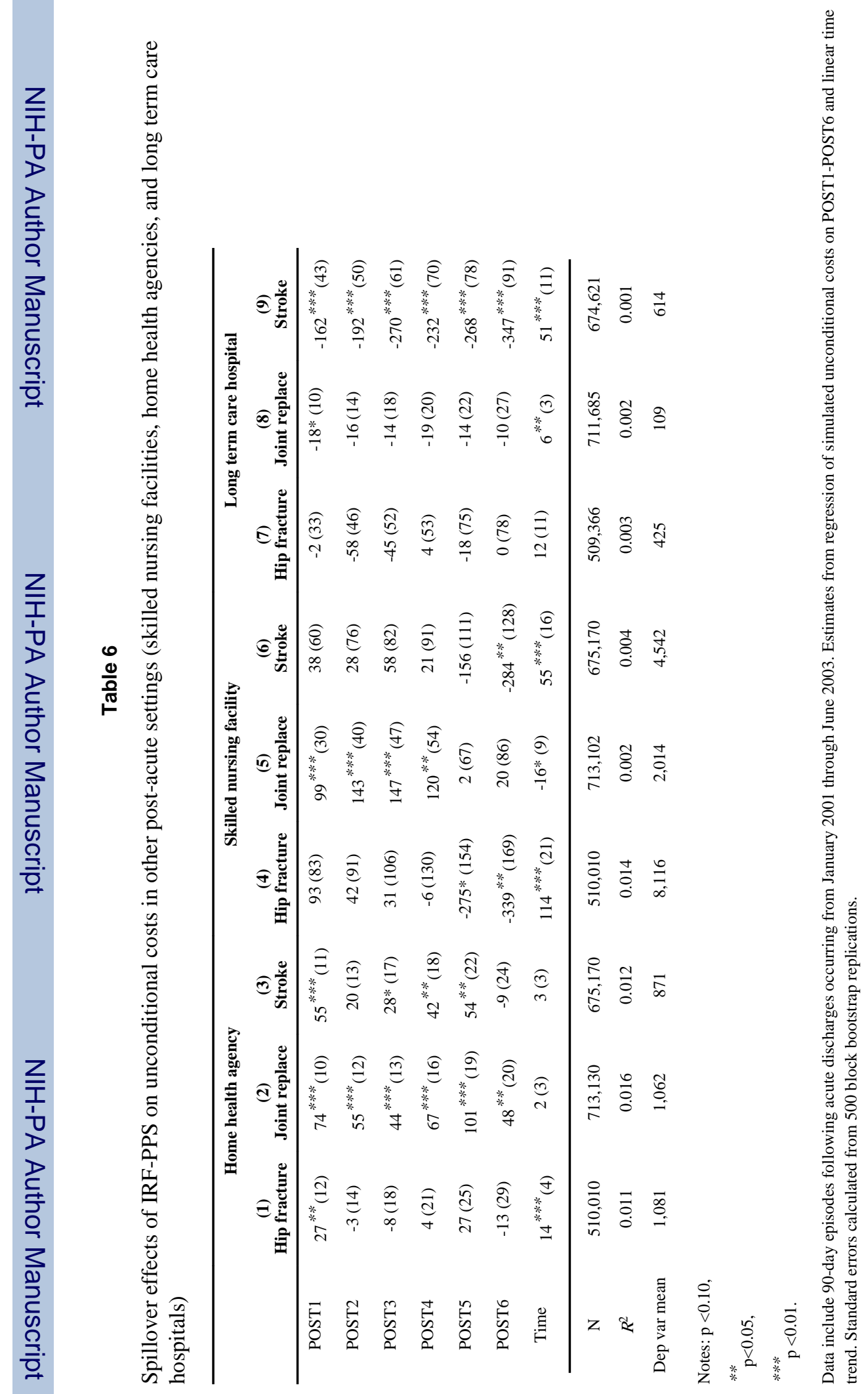




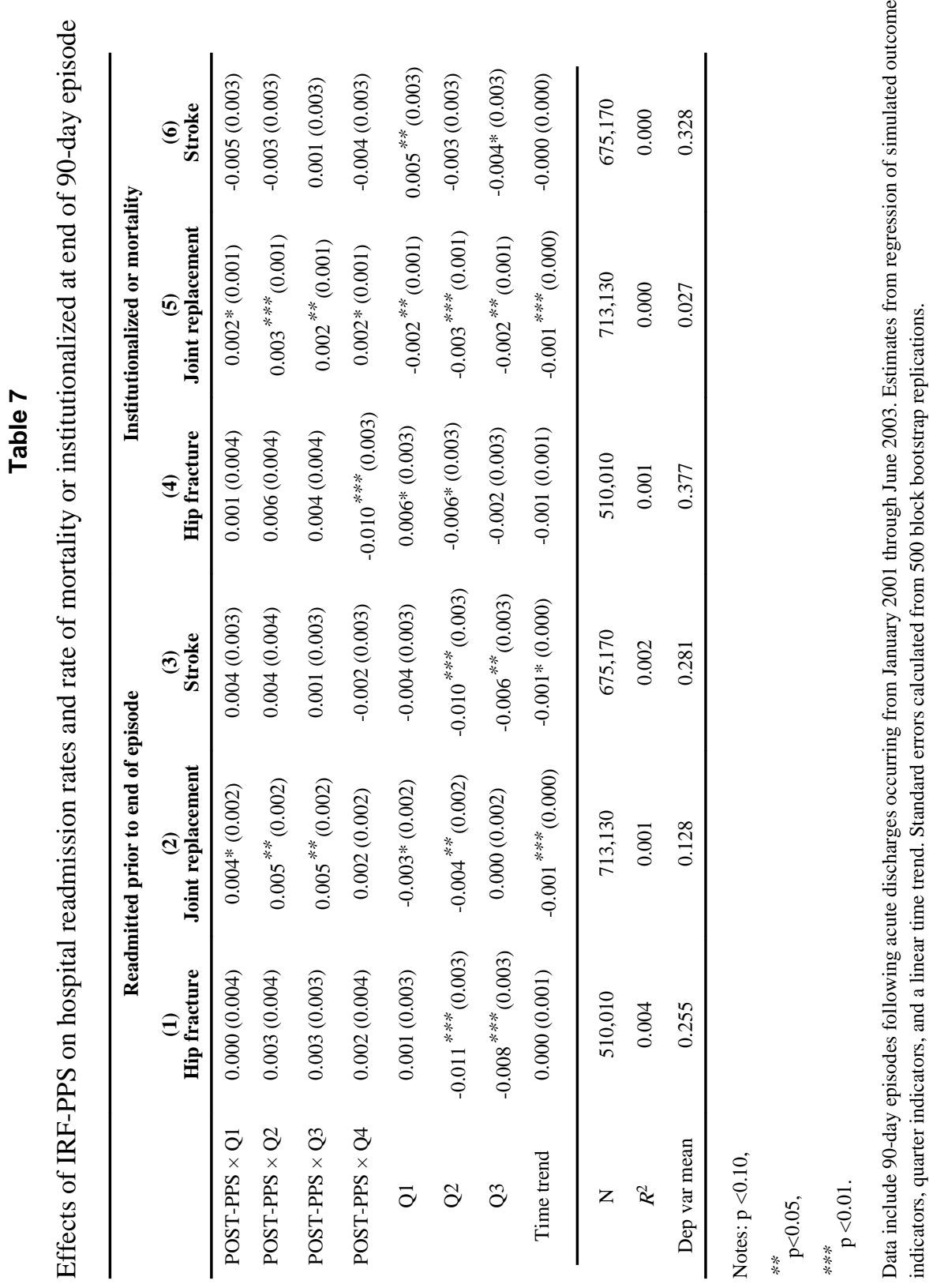




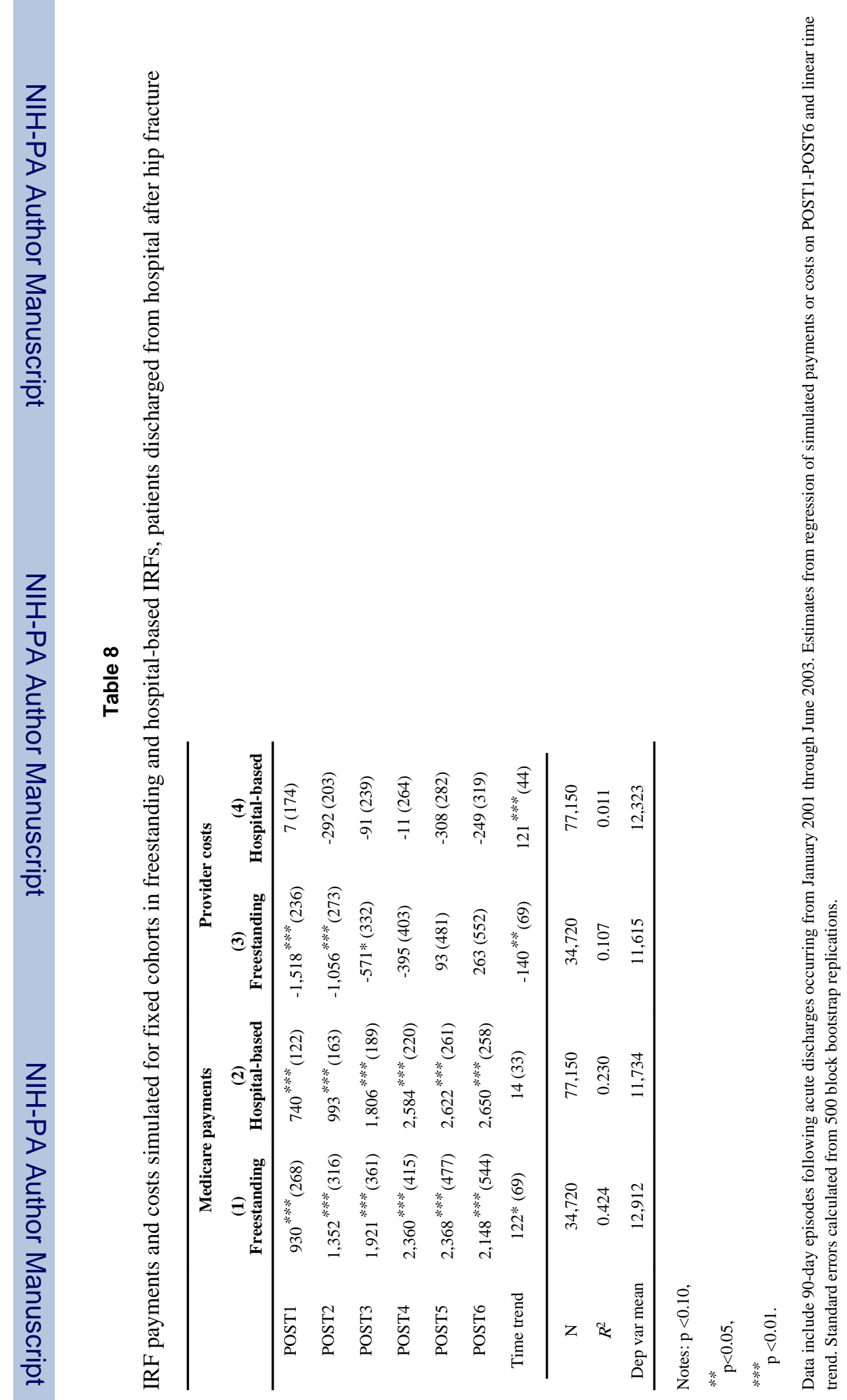

$J$ Health Econ. Author manuscript; available in PMC 2014 September 01. 


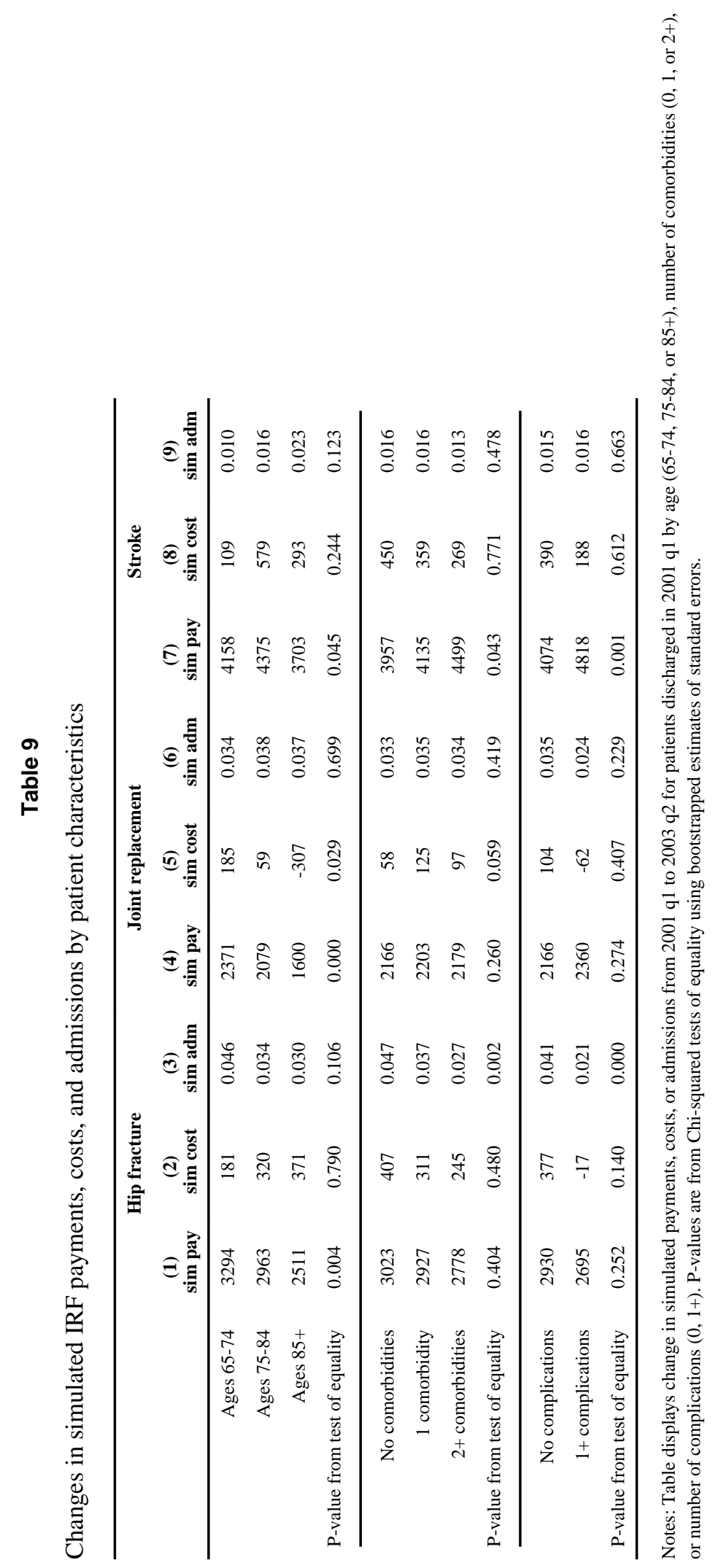

